\title{
The effects of disturbances in non-iterative data-driven controller design
}

\author{
Eduardo V. da Silva* Luciola Campestrini * \\ * Departamento de Sistemas Elétricos de Automação e Energia, \\ Universidade Federal do Rio Grande do Sul, Brasil \\ (email: $\{$ eduardo.varriale,luciola\}@ufrgs.br).
}

\begin{abstract}
Non-iterative data-driven control design techniques are very compelling for their ease of implementation and the convenience of only using one set of collected data to do the design. During the period of the data set collection, disturbances that may have occurred to the plant will influence on the design results. On this paper, we use a refrigeration system model to test the effects of different disturbance signals during a closed-loop test to the performance of the controllers obtained through two different non-iterative data-driven controller design methods. The controllers obtained were compared on how far the performance obtained was from the desired response and on the Ratio of Integrated Absolute Variation of Control signal (RIAVU). The results show that the disturbances do interfere in the estimated controllers, changing the closed-loop response. Filtering the collected data before applying the data-driven methodologies improved the results significantly, considering the chosen disturbance signals.
\end{abstract}

\section{Keywords: Data-driven Controller Design, Control Algorithms, Disturbances, OCI, VRFT.}

\section{INTRODUCTION}

Data-driven controller design offers alternative methods for designing a controller when the model of the plant one wants to control is not available. Using collected data from an experiment on the system, a fixed-structure controller is designed in order to obtain a closed-loop system as close as possible to a desired response (Bazanella et al., 2012). This is very useful in practice, since the model of the plant is often not available or too complex for controller design purposes and creating new or simplified models can be costly and time-consuming.

Among several data-driven control techniques, the noniterative ones (also known as one-shot) have been largely applied to real systems due to the fact that only one batch of input-output data is required in order to design the controller. Virtual Reference Feedback Tuning (VRFT) (Campi et al., 2000), Correlation-based Tuning (CbT) (Karimi et al., 2007) and the Optimal Controller Identification (OCI) (Campestrini et al., 2017) are representative of such group. Even though data can be obtained in open or closed-loop experiments, the majority of data-driven control literature presents practical results using openloop data (Bazanella et al., 2012; Formentin et al., 2013; Rojas et al., 2011; Campestrini et al., 2017). However, with the growth of historic data availability, the use of closedloop data, collected directly from historic data becomes of great interest, specially because it is not necessary to add disturbances to the process or to open the control loop, which could result in production losses and safety concerns.

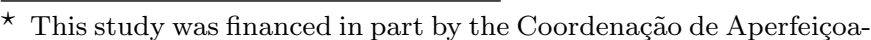
mento de Pessoal de Nível Superior - Brasil (CAPES) - Finance Code 001.
}

When data is collected from historic data sets, it is often the case such data is affected by unknown disturbances, which may interfere in the control design. Besides, even when opening the loop to perform an experiment, the collected signals can be affected by disturbances and the experiment could be compromised. Some work has been done on defining sufficiently rich signals (Gevers et al., 2009) and on finding informative interval data records (Arengas and Kroll, 2017) for system identification, but a lot less research can be found about the effects that disturbances have on the controllers obtained by datadriven methods.

On this paper, the effect of the disturbances in the collected data is tackled, considering the controllers are designed using closed-loop data and two different methodologies: VRFT and OCI. Using a rather complex model of a refrigeration system, presented as a Benchmark for a PID competition (Bejarano et al., 2017), held at the PID18 conference, a controller will be estimated with different data sets, affected with different disturbances. Considering collected data without disturbances, a controller is obtained with each methodology to be used for comparison. By generating new data sets with different disturbances, the changes on the estimated controllers and in the closedloop responses are analyzed. The quality of a controller is measured by the difference between the closed-loop response obtained and desired response.

In Section 2 the control design formulation is presented for both applied methodologies: VRFT and OCI. Section 3 , presents the plant we use for analyzing the influence of disturbances on the collected data. Next, in Section 4 we show the experimental setup and the resulting controllers, while Section 5 presents the analysis of the results. Finally, 
we present some conclusions and the proposed future work on these subject.

\section{ONE-SHOT DATA-DRIVEN CONTROL}

Consider a linear time-invariant discrete-time SISO process

$$
y(k)=G(q) u(k)+v(k),
$$

where $q$ is the forward-shift operator, $G(q)$ represents the process, $u(k)$ is the control input and $v(k)=H(q) e(k)$ represents the noise, which is white noise $e(k)$ filtered by $H(q)$. We want to design the parameter vector $\rho \in \Re^{p}$ of a linear-time invariant controller $C(q, \rho)$ such that the closed-loop system becomes as close as possible to the performance specified through a desired closed-loop transfer function $T_{d}(q)$, also known as the reference model. The control action $u(k)$ can be written as

$$
u(k)=C(q, \rho)(r(k)-y(k)),
$$

where $r(k)$ is the reference signal, which is assumed to be uncorrelated with the noise. In closed loop, the system given by (1) and (2) becomes

$$
y(k, \rho)=T(q, \rho) r(k)+S(q, \rho) v(k),
$$

where $S(q, \rho)=(G(q) C(q, \rho)+1)^{-1}$ and $T(q, \rho)=$ $S(q, \rho) G(q) C(q, \rho)$.

The optimal parameter $\rho$ of the controller can be found by solving the model reference (MR) optimization problem

$$
\begin{gathered}
\hat{\rho}=\arg \min J^{M R}(\rho), \\
J^{M R}(\rho)=\sum_{k=1}^{N}\left[\left(T_{d}(q)-T(q, \rho)\right) r(k)\right]^{2} .
\end{gathered}
$$

The ideal controller is the one that results in a cost $J^{M R}$ of 0 and is given by

$$
C_{d}(q)=\frac{T_{d}(q)}{G(q)\left(1-T_{d}(q)\right)},
$$

Notice that (5) depends on the knowledge of $G(q)$. Datadriven control methods solve the model reference problem using data collected from an experiment, without using a mathematical model for the process. Besides the collected data, data-driven design needs the definition of the desired closed-loop response, given by $T_{d}(q)$ and the controller class to be identified. Different controller structures can be identified, however in this paper we are going to design PI controllers, which have a linear parametrization, i.e. can be written as

$$
C(q, \rho)=\rho^{T} \bar{C}(q),
$$

where $\bar{C}(q)$ is an $p$-vector of fixed causal rational functions. The PI controller can be written as

$$
C(q, \rho)=\left[\begin{array}{ll}
P & I
\end{array}\right]\left[\begin{array}{c}
1 \\
\frac{1}{q-1}
\end{array}\right]
$$

Both virtual reference feedback tuning (VRFT) and optimal controller identification (OCI) are one-shot techniques that may be used to solve the model reference optimization problem. They are briefly presented in the sequel.

For both methods, consider that an open or closed-loop experiment is performed resulting in the collected input/output data:

$$
Z=\{u(k), y(k), \quad \text { for } \quad k=1, \ldots, n\} .
$$

\subsection{Virtual reference feedback tuning}

The VRFT method was first introduced in Campi et al. (2000) and has been extended to solve important problems as the control of NMP systems (Bazanella et al., 2012) and MIMO systems (Campestrini et al., 2016; Goncalves da Silva et al., 2018) as well as to different control strategies (Lecchini et al., 2001; Eckhard et al., 2018). Besides, it has been widely applied to real systems (Campestrini et al., 2016; Rojas et al., 2010).

Its success can be explained by the fact that it uses only one batch of input-output data, collected in open or closed loop, and solves a quadratic problem. The idea of the method can be explained as follows: from the collected output $y(k)$, the virtual reference is calculated as $\bar{r}(k)=T_{d}^{-1}(q) y(k)$, which is the reference signal that would result in $y(k)$ if the ideal controller were in the loop. The virtual error is then given by $\bar{e}(k)=\bar{r}(k)-$ $y(k)$. So, the ideal controller presents the collected $u(k)$ as output when fed by $\bar{e}(k)$, turning the problem into an identification problem. To solve this problem, VRFT minimizes the following criterion:

$$
\begin{gathered}
\hat{\rho}=\arg \min J^{V R}(\rho) \\
J^{V R}(\rho)=\sum_{k=1}^{n}[L(q)(u(k)-C(q, \rho) \bar{e}(k))]^{2},
\end{gathered}
$$

where $L(q)$ is a filter used to approximate the minima of (4) and (10) when the ideal controller $C_{d}(q)$ does not belong to the chosen controller class. The filter $L(q)$ should be chosen such that

$$
\left|L\left(e^{j \omega}\right)\right|^{2}=\left|T_{d}\left(e^{j \omega}\right)\right|^{2}\left|S\left(e^{j \omega}, \rho\right)\right|^{2} \frac{\Phi_{r}\left(e^{j \omega}\right)}{\Phi_{u}\left(e^{j \omega}\right)}, \forall \omega \in[-\pi, \pi]
$$

where $\Phi_{r}\left(e^{j \omega}\right)$ and $\Phi_{u}\left(e^{j \omega}\right)$ represent the spectrum of the reference we want to apply to the closed-loop system and the input of the test, respectively. From (12) we see that there is a dependence on the unknown parameter vector $\rho$ and on the spectrum of the applied input signal $u(k)$. It is often the case the filter is approximated by

$$
L(q)=T_{d}(q)\left(1-T_{d}(q)\right) .
$$

Notice that besides approximating the minima (5) and (11), this filter attenuates the influence of high-frequency noise (by $T_{d}(q)$ ) and DC components (by $\left.1-T_{d}(q)\right)$. It plays an important role when dealing with data corrupted by noise and/or step disturbances.

The optimization (10) is solved by computing:

$$
\begin{gathered}
\bar{\varphi}(k)=\bar{C}(q)\left(T_{d}(q)^{-1}-1\right) L(q) y(k) \\
\hat{\rho}=\left[\sum_{k=1}^{n} \bar{\varphi}(k) \bar{\varphi}(k)\right]^{-1} \sum_{k=1}^{n} \bar{\varphi}(k) L(q) u(k) .
\end{gathered}
$$

\subsection{Optimal Controller Identification}

The Optimal Controller Identification method was presented in Campestrini et al. (2017) and extended to MIMO systems in Huff et al. (2019), both presenting applications to real systems. Different from VRFT, which identifies the ideal controller through least squares, the OCI method identifies it through the prediction error approach. By doing so, the statistical properties of the estimate are 
enhanced compared to VRFT when data is corrupted with noise.

Consider we want to identify a parametrized model for the system given by (1). Since we want to identify the ideal controller (6), the plant can be written as

$$
G(q)=\frac{1}{C_{d}(q)} \frac{T_{d}(q)}{\left(1-T_{d}(q)\right)} .
$$

So, a parametrized model for the plant can be written in terms of the parametrized controller, to be identified, and the reference model, defined by the user

$$
G(q, \rho)=\frac{1}{C(q, \rho)} \frac{T_{d}(q)}{\left(1-T_{d}(q)\right)} .
$$

Also, it is often the case that the controller has a fixed part (as the integrator in PI controllers), which does not need to be identified. The controller can then be written as $C(q, \rho)=C^{I}(q, \rho) C^{F}(q)$, where $C^{F}(q)$ is the fixed part and $C^{I}(q, \rho)$ is the part to be identified.

Defining

$$
\tilde{C}(q, \rho)=\frac{1}{C^{I}(q, \rho)}=\frac{C^{F}(q)}{C(q, \rho)},
$$

the systems input-output model can be written as

$$
\begin{aligned}
y(k, \theta) & =\underbrace{\frac{1}{C^{I}(q, \rho)}}_{\tilde{C}(q, \rho)} \times \underbrace{\frac{T_{d}(q)}{C^{F}(q)\left(1-T_{d}(q)\right)} u(k)}_{\tilde{u}(k)}+H(q, \theta) e(k) \\
& =\tilde{C}(q, \rho) \tilde{u}(k)+H(q, \theta) e(k),
\end{aligned}
$$

where $\theta=\left[\begin{array}{ll}\rho^{T} & \eta^{T}\end{array}\right]^{T}$ and $\eta \in \Re^{c}$ is an additional parameter vector which appears in the noise model. The method solves the following criterion:

$$
\begin{gathered}
\hat{\theta}=\arg \min J^{O C I}(\theta) \\
J^{O C I}(\theta)=\sum_{k=1}^{n}[y(k)-\hat{y}(k \mid k-1, \theta)]^{2},
\end{gathered}
$$

where $\hat{y}(k \mid k-1, \theta)$ is the optimal one-step-ahead predictor for model (19):

$\hat{y}(k \mid k-1, \theta)=H^{-1}(q, \theta) \tilde{C}(q, \rho) \tilde{u}(k)+\left[1+H^{-1}(q, \theta)\right] y(k)$

The chosen structure for the noise model will result in different model structures and hence different algorithms to solve such problem. However, commercial tools as MATLAB ${ }^{\circledR}$ ident can be used to design such controllers.

\section{CASE STUDY}

As mentioned above, both VRFT and OCI are one-shot methods, that is, perform the design of the controller based on only one batch of input-output data collected from the plant. Even if these data can be collected in open or closed loop, it is often the case the results presented in datadriven control literature are based on open-loop data. In this paper, we want to test the application of both methods using data collected in closed-loop experiments.

Besides that, it is often the case the process variables are affected by different kinds of disturbances during operation and we want to test how much such disturbances interfere in the controller design. For that reason, we use a rather complex problem to generate such data, presented as a benchmark to a competition in a conference on PID control (Bejarano et al., 2017). This system was chosen for being a nonlinear plant which has several disturbance inputs available and comes with its control-loop closed by simple controllers. It is a canonical one-compressionstage, one-load-demand refrigeration cycle as shown in Fig. 1, where the main components (the expansion valve, the compressor, the evaporator, and the condenser) are represented. The objective is to cool the secondary flux at the evaporator, by removing heat, and rejecting this heat to the secondary flux at the condenser.

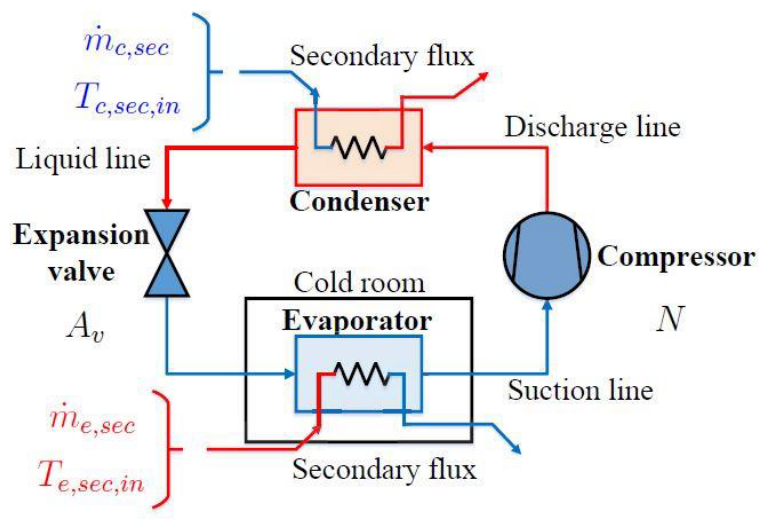

Figure 1. Schematic picture of one-compression-stage, oneload-demand vapor-compression refrigeration cycle (Bejarano et al., 2017)

The control problem proposed by the benchmark is a MIMO system where the outlet temperature of the evaporator secondary flux $T_{e, s e c, o u t}$ and the degree of superheating of the refrigerant at the evaporator outlet $T_{S H}$ are meant to be controlled by manipulating the compressor speed $N$ and the expansion valve opening $A_{v}$. It comes with a decentralized pair of controllers, dealing with each pair of variables as SISO systems. The controller

$$
C_{1}(q)=\frac{-1.0136(q-1.024)(q+0.9623)}{(q-1)(q-0.9853)}
$$

is used to control the $T_{e, s e c, o u t}$, manipulating $N$. To control the $T_{S H}$, manipulating $A_{v}$, the controller

$$
C_{2}(q)=\frac{0.42 q-0.02}{q-1}
$$

is used. The system also presents several other variables that act as immeasurable disturbances. These disturbances are presented in Table 1 and affect the controlled variables through changes on the operation point and characteristics of the plant.

Table 1. Disturbances supported by the model

\begin{tabular}{ccc} 
Disturbance & Symbol & Unit \\
\hline Inlet temperature of the condenser secondary flux & $T_{c, s e c, i n}$ & ${ }^{\circ} \mathrm{C}$ \\
Mass flow of the condenser secondary flux & $\dot{m}_{c, s e c}$ & $\mathrm{~g} / \mathrm{s}$ \\
Inlet pressure of the condenser secondary flux & $P_{c, s e c, i n}$ & $\mathrm{bar}$ \\
Inlet temperature of the evaporator secondary flux & $T_{e, s e c, i n}$ & ${ }^{\circ} \mathrm{C}$ \\
Mass flow of the evaporator secondary flux & $\dot{m}_{e, s e c}$ & $\mathrm{~g} / \mathrm{s}$ \\
Inlet pressure of the evaporator secondary flux & $P_{e, s e c, i n}$ & $\mathrm{bar}$ \\
Compressor surroundings temperature & $T_{s u r r}$ & ${ }^{\circ} \mathrm{C}$ \\
\hline
\end{tabular}

In this paper, the controller $C_{1}(q)$ will be redesigned through both VRFT and OCI methods, using closed-loop data corrupted by different disturbance signals applied to 
the $T_{c, s e c, \text { in }}$ variable to evaluate the effect of disturbances in data-driven control methods. By considering the redesign only of one controller, the other control loop is seen as part of the plant and also acts as a disturbance to the loop.

\section{EXPERIMENTS AND CONTROLLER DESIGN}

In order to compare the influence of disturbances in the collected data applied to data-driven control, the following experimental setup was considered: the refrigeration system is in closed loop with (22) and (23); while the reference signal of $T_{S H}$ is kept constant at value 14.65 , the signal presented in Fig. 2 is applied as reference signal to $T_{e, \text { sec,out, }}$ which is a sum of square waves with different periods and delays and amplitude between $-22.15{ }^{\circ} \mathrm{C}$ and $-22.65^{\circ}$, the same amplitude as the signal used in Bejarano et al. (2017).

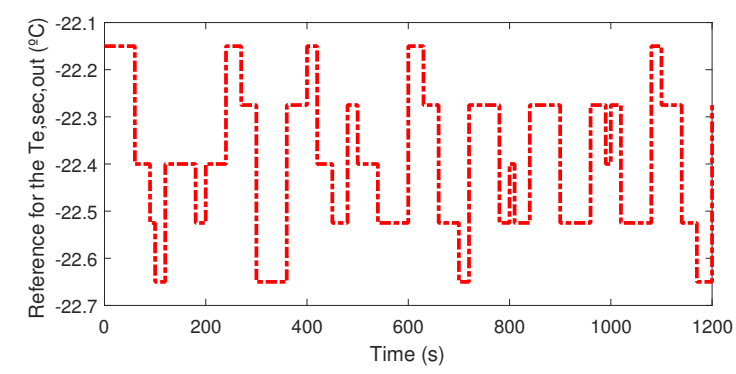

Figure 2. Reference signal for the temperature on the output of the secondary flux of the evaporator.

Several tests are performed, considering different disturbances' occurrences. All simulations were ran until the $1200 \mathrm{~s}$ mark, with a sample time $\left(T_{s}\right)$ of $0.1 \mathrm{~s}$. During the first test ("No Disturbance"), all disturbance variables are kept constant at their nominal value. During the other tests, different inlet temperature of the condenser secondary flux signal were considered. On the second data set ("Step at $960 \mathrm{~s}$ ") the default disturbance signal proposed by the PID Benchmark for this variable was used, which is a step form $30{ }^{\circ} \mathrm{C}$ to $27^{\circ} \mathrm{C}$, at $960 \mathrm{~s}$. A set of tests ("Step at $600 \mathrm{~s}$ ", "Step at $300 \mathrm{~s}$ ", "Step at $0 \mathrm{~s}$ ") were ran using a step function as disturbance, much like the one from the second test, but with the step time at $600 \mathrm{~s}, 300 \mathrm{~s}$ and $0 \mathrm{~s}$ respectively. The sixth test ("Sum of Square Signals") uses a sum of square signals for the disturbance, similar to the one used in the reference, but with amplitudes adjusted to be within the same 3 degrees as the original, centered in the $30{ }^{\circ} \mathrm{C}$ and with different period to guarantee no correlation with the reference signal. The seventh data set ("White Noise") is taken using a white noise of mean $30{ }^{\circ} \mathrm{C}$ and variance of $0.25^{\circ} \mathrm{C}$. Figure 3 presents the effect that the step disturbance at $600 \mathrm{~s}$ causes in the controlled variable $T_{e, s e c, \text { out }}$ and in the manipulated variable $A_{v}$. The controller used in the tests rejects the disturbance and there is little difference between $T_{e, s e c, o u t}$ with and without disturbance, but a clear effect can be seen in $A_{v}$.

As the focus of this paper is the effect of the disturbances on the controllers and not the search for the best controller possible, simple reference model and controller class will
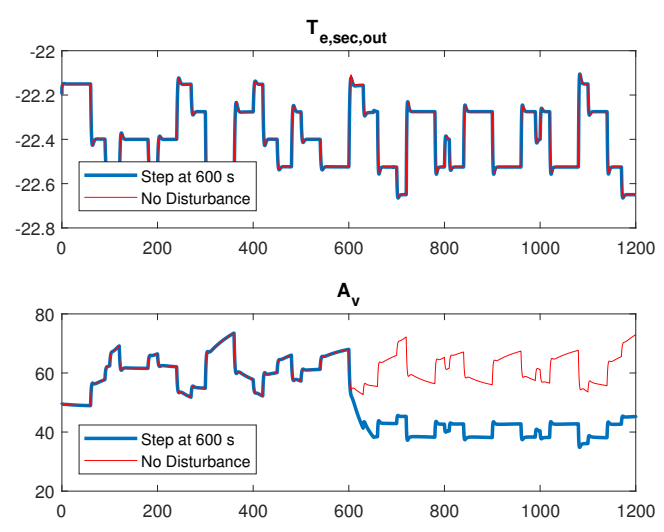

Figure 3. Comparison of data sets collected with and without a step disturbance.

be used. The reference model is defined as a first order transfer function with DC gain equal to 1 ,

$$
T_{d}(q)=\frac{0.1}{q-0.9},
$$

and the controller class used is the one presented in (8). The controllers obtained with data-set "No disturbance" are considered the best controller within the defined controller class and chosen methodology, and are used to evaluate how bad is the influence of the disturbance.

The data sets, reference model and controller class were used to calculate the parameters $P$ and $I$ of the controller through both VRFT and OCI approaches. Since the VRFT filter $L(q)$ presented in (13) removes lower and higher frequencies, its application is fundamental when the collected signals are corrupted with disturbances. Figure 4 shows the same collected signal as Figure 3, but filtered by $L(q)$. Notice that, since the controller is able to reject such disturbance, its influence appears mainly on the control variable. However, after filtering signals, the disturbance effect is highly attenuated.
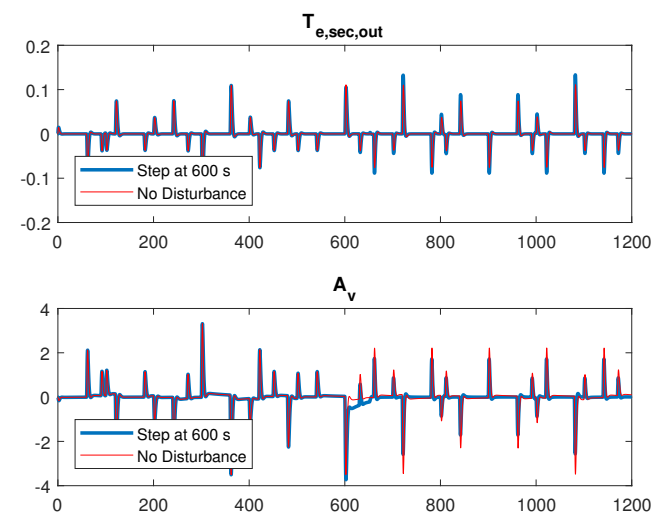

Figure 4. Comparison of data sets collected with and without a step disturbance, to be used in the control design, after filtering by $L(q)$.

It is important to highlight here that the OCI formulation does not consider any filtering of the signals, but when the chosen controller class is such that the ideal controller does not belong to it, the minimum of (21) is different from the 
Table 2. PI controller parameters estimated by the OCI approach and unfiltered data.

\begin{tabular}{ccc} 
Disturbance & $\mathrm{P}$ & $\mathrm{I}$ \\
\hline No Disturbance & -2.5557 & -5.1058 \\
Step at $960 \mathrm{~s}$ & -26707.4 & -4.4109 \\
Step at $600 \mathrm{~s}$ & -69517.1 & -8.1318 \\
Step at $300 \mathrm{~s}$ & 99.96 & 138.1346 \\
Step at $0 \mathrm{~s}$ & 12.03 & 17.8333 \\
Sum of Square Signals & 6715.3 & 1.4074 \\
White Noise & 2641.7 & 1.5634 \\
\hline
\end{tabular}

Table 3. PI controller parameters estimated by the VRFT approach with different data sets

\begin{tabular}{ccc} 
Disturbance & $\mathrm{P}$ & $\mathrm{I}$ \\
\hline No Disturbance & -1.2804 & -3.0429 \\
Step at $960 \mathrm{~s}$ & -1.4207 & -2.8282 \\
Step at $600 \mathrm{~s}$ & -1.8635 & -2.4588 \\
Step at $300 \mathrm{~s}$ & -2.4753 & -2.1252 \\
Step at $0 \mathrm{~s}$ & -2.3972 & -1.9199 \\
Sum of Square Signals & -1.1937 & -2.9882 \\
White Noise & -1.3557 & -3.0417
\end{tabular}

Table 4. PI controller parameters estimated by the OCI approach with different data sets

\begin{tabular}{ccc} 
Disturbance & $\mathrm{P}$ & $\mathrm{I}$ \\
\hline No Disturbance & -7.3083 & -3.0931 \\
Step at $960 \mathrm{~s}$ & -7.2157 & -3.0233 \\
Step at $600 \mathrm{~s}$ & -7.0952 & -2.7164 \\
Step at $300 \mathrm{~s}$ & -6.9944 & -2.2395 \\
Step at 0 s & -6.3065 & -1.9616 \\
Sum of Square Signals & -1.9235 & -3.3347 \\
White Noise & -7.3927 & -3.0953 \\
\hline
\end{tabular}

minimum of (5). When we apply OCI using data without filtering them, we obtain the results presented in Table 2. Notice that the controller parameters are completely different using each data set, and some of them would probably make the closed loop unstable.

So, we collect input and output data, filter them by (13) and apply both VRFT and OCI. The resulting controller parameters for each data set are presented in Table 3 for VRFT and Table 4 for OCI.

\section{ANALYSIS OF RESULTS}

Using the same $1200 \mathrm{~s}$ duration and sample time of $0.1 \mathrm{~s}$ as the previous experiments, the system was simulated with the default reference signal and all the disturbances included in the benchmark PID 2018 (Bejarano et al., 2017), for each one of the calculated controllers. The default reference signal is a step from -22.15 to $-22.65{ }^{\circ} \mathrm{C}$ at $120 \mathrm{~s}$, and the main disturbances are the reference for $T_{S H}, T_{c, s e c, i n}$ and $T_{e, s e c, i n}$, presented in Figure 5 . To evaluate how much the controller design was affected by the disturbances present on collected data, the results were compared based on two different indices.

A ratio that represents the mean deviation from the desired response per sample. This index is relative, so all the controllers obtained by each method will be compared with the one obtained through the same methodology using signals without disturbances as the baseline. It is described by

$$
R J\left(C_{1}, C_{2}\right)=\frac{J\left(C_{2}\right)}{J\left(C_{1}\right)}
$$

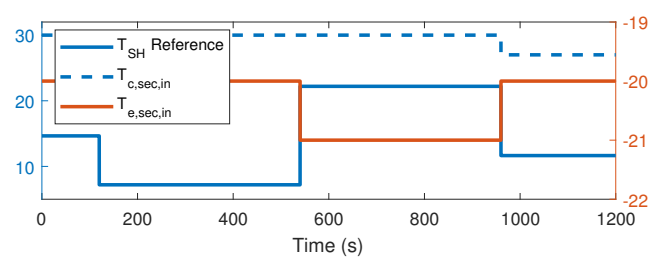

Figure 5. Main disturbances applied during controller analysis experiments

where,

$$
J(C)=\sqrt{\sum_{k=n_{1}}^{n_{2}} \frac{\left(y_{C}(k)-y_{R M}(k)\right)^{2}}{n_{2}-n_{1}}},
$$

$C_{1}$ is the baseline controller, $C_{2}$ is the controller being compared to the baseline, $y_{C}$ is $T_{e, s e c, o u t}$ (outlet temperature of the evaporator secondary flux), $y_{R M}$ is the desired response considering the defined reference model (24), and $n_{1}$ and $n_{2}$ are the first and last samples of the period considered in the calculation of the index. This index is calculated using the data from the time of the step change on reference until nine seconds later, when both closed loops have settled on the reference value.

The controllers were also evaluated based on a performance index, suggested by Bejarano et al. (2017): the Ratio of Integrated Absolute Variation of Control signal (RIAVU). It is also a relative index and is described by

$$
R I A V U\left(C_{1}, C_{2}\right)=\frac{I A V U\left(C_{2}\right)}{I A V U\left(C_{1}\right)}
$$

where

$$
I A V U=\sum_{k=2}^{n}|u(k)-u(k-1)| T_{s},
$$

and $u(k)$ is the control signal. RIAVU compares how much the control signal varies through the experiment, representing the effort of the actuator.

Tables 5 and 6 present the indices relative to each estimated controller. As can be seen, every disturbance affected the performance of the controller at different degrees. From the step disturbances, in both methods, it can be observed that the higher the amount of time the data was affected by the disturbance, the further from 1 was the resulting RJ of the controller. When comparing the disturbances that affected the whole data (Step at 0 s, Sum of Square Signals and White Noise) it can be inferred that the higher the variance of the disturbance signal to the nominal value, the greater the effect on the RJ index in both methods. The fact that, in some cases, having a disturbance may end up granting a better result than otherwise reminds us of the stochastic nature of the interference of disturbances.

Another thing we can observe from the results is that the VRFT method was less affected by the disturbances, resulting on designs closer to the one using data without disturbance, on average. The scope of this paper does not include the comparison between the efficiency of both methods, but, as a side note, when comparing them, OCI resulted in lower cost $J$ with every data-set, except for the one affected by the Sum of Square Signals. The parameters obtained by OCI using data without disturbances and without applying filter $L(q)$ (see Table 2) resulted in a 
Table 5. Performance index of the different controllers obtained by VRFT approach

\begin{tabular}{cccc} 
Disturbance & $\mathrm{J}$ & RJ & RIAVU \\
\hline No Disturbance & 0.0157 & 1 & 1 \\
Step at $960 \mathrm{~s}$ & 0.0137 & 0.8718 & 0.9981 \\
Step at $600 \mathrm{~s}$ & 0.0178 & 1.1301 & 0.9946 \\
Step at $300 \mathrm{~s}$ & 0.0290 & 1.8431 & 0.9912 \\
Step at 0 s & 0.0388 & 2.4682 & 0.9891 \\
Sum of Square Signals & 0.0153 & 0.9755 & 0.9996 \\
White Noise & 0.0155 & 0.9857 & 0.9998 \\
\hline
\end{tabular}

Table 6. Performance index of different controllers obtained through OCI approach

\begin{tabular}{cccc} 
Disturbance & $\mathrm{J}$ & RJ & RIAVU \\
\hline No Disturbance & 0.0111 & 1 & 1 \\
Step at $960 \mathrm{~s}$ & 0.0100 & 0.8995 & 0.9995 \\
Step at 600 s & 0.0097 & 0.8745 & 0.9979 \\
Step at 300 s & 0.0233 & 2.1008 & 0.9956 \\
Step at 0 s & 0.0353 & 3.1760 & 0.9916 \\
Sum of Square Signals & 0.0191 & 1.7209 & 1.0065 \\
White Noise & 0.0112 & 1.0112 & 1.0000 \\
\hline
\end{tabular}

cost $J=0.0482{ }^{\circ} \mathrm{C}$, showing the importance of the use of such a filter in the case where the chosen controller is underparametrized. On the other hand, the $R I A V U$ index shows that the control cost was barely affected by the disturbances.

Figure 6 show the results of the best and worse controllers calculated through the OCI method. It also shows the reference signal and the expected result, described by the behavior of the reference model (24) to the reference signal.

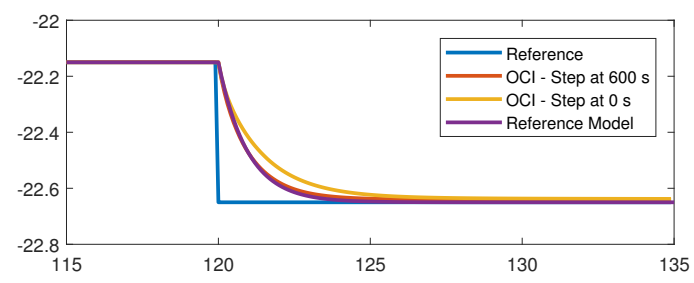

Figure 6. Comparison of the closed loop obtained with two controllers calculated through OCI and the desired response.

\section{CONCLUSIONS}

This paper focused on evaluating the effects of disturbances on the resulting controller obtained by one-shot data-driven methods. Using data from closed loop experiments, the effect of step or noise disturbances was evaluated. These disturbance signals, typical to this type of system, had a substantial effect on the controller performance. Using the $L(q)$ filter, given by (13), this effect was greatly diminished, estimating controllers close to the one estimated without disturbances.

For future work, it would be interesting to define a more appropriate filter to be used for such purpose. The one given by (13) would probably not be so efficient for other disturbances, as sinusoidal signals with frequencies in the reference model passing band, for example.

\section{REFERENCES}

Arengas, D. and Kroll, A. (2017). Searching for informative intervals in predominantly stationary data records to support system identification. ICAT 2017 - 26th International Conference on Information, Communication and Automation Technologies, Proceedings, 2017Decem, 1-6.

Bazanella, A.S., Campestrini, L., and Eckhard, D. (2012). Data-Driven Control Design: The H2 Approach. Springer.

Bejarano, G., Alfaya, J.A., Rodríguez, D., Ortega, M.G., and Morilla, F. (2017). Benchmark for PID control of refrigeration systems based on vapour compression.

Campestrini, L., Eckhard, D., Chía, L.A., and Boeira, E. (2016). Unbiased MIMO VRFT with application to process control. Journal of Process Control, 39, 35-49.

Campestrini, L., Eckhard, D., Sanfelice Bazanella, A., and Gevers, M. (2017). Data-driven model reference control design by prediction error identification. Journal of the Franklin Institute, 354(6), 2628-2647.

Campi, M., Lecchini, a., and Savaresi, S. (2000). Virtual reference feedback tuning (VRFT): a new direct approach to the design of feedback controllers. Proceedings of the 39th IEEE Conference on Decision and Control (Cat. No.00CH37187), 1(1), 1337-1346.

Eckhard, D., Campestrini, L., and Christ Boeira, E. (2018). Virtual disturbance feedback tuning. IFAC Journal of Systems and Control, 3(February), 23-29.

Formentin, S., Filippi, P.D., Corno, M., Tanelli, M., and Savaresi, S.M. (2013). Data-driven design of braking control systems. IEEE Transactions on Control Systems Technology, 21(1), 186-193.

Gevers, M., Bazanella, A.S., Bombois, X., and Mišković, L. (2009). Identification and the information matrix: How to get just sufficiently rich? IEEE Transactions on Automatic Control, 54(12), 2828-2840.

Goncalves da Silva, G.R., Campestrini, L., and Bazanella, A.S. (2018). Multivariable Virtual Reference Feedback Tuning for Non-Minimum Phase Plants. IEEE Control Systems Letters, 2(1), 121-126.

Huff, D., Campestrini, L., R. Gonçalves da Silva, G., and S. Bazanella, A. (2019). Data-driven control design by prediction error identification for multivariable systems. Journal of Control, Automation and Electrical Systems.

Karimi, A., van Heusden, K., and Bonvin, D. (2007). Noniterative data-driven controller tuning using the correlation approach. In 2007 European Control Conference (ECC), 5189-5195.

Lecchini, A., Campi, M.C., and Savaresi, S.M. (2001). Sensitivity shaping via virtual reference feedback tuning. Proceedings of the IEEE Conference on Decision and Control, 1(December), 750-755.

Rojas, J.D., Baeza, J.A., and Vilanova, R. (2011). Three degrees of freedom virtual reference feedback tuning design and its application to wastewater treatment plant control. In Proceedings of 18th IFAC World Congress, volume 44, 7144-7149. 18th IFAC World Congress.

Rojas, J.D., Tadeo, F., and Vilanova, R. (2010). Control of a ph neutralization plant using the vrft framework. In 2010 IEEE International Conference on Control Applications, 926-931. 